\title{
Damage from Proton Irradiation of Vertical-Cavity Surface-Emitting Lasers ${ }^{1}$
}

\author{
Alan H. Paxton ${ }^{2}$, Member, IEEE, Richard F. Carson ${ }^{3}, M e m b e r, I E E E$, Harald Schöne ${ }^{2}$, \\ Edward W. Taylor ${ }^{2}$, Member, IEEE, Kent D. Choquette ${ }^{3}$, Member, IEEE, \\ Hong Q. Hou ${ }^{3}$, Member, IEEE, Kevin L. Lear ${ }^{3}$, Member, IEEE, and Mial E. Warren ${ }^{3}$ \\ ${ }^{2}$ U.S. Air Force Phillips Laboratory/VTMC, Kirtland AFB, NM 87117-5776 \\ ${ }^{3}$ Sandia National Laboratories, MS 0603, Albuquerque, NM 87185-1056
}

\begin{abstract}
Damage resulting from irradiating oxide-confined verticalcavity surface-emitting lasers became significant (threshold shift $\approx 20 \%$, peak power degradation $\approx 20 \%$ ) at fluence levels approaching $1 \times 10^{13}$ protons $/ \mathrm{cm}^{2}$. The threshold current shifted to higher values, and the peak light output power decreased. Forward-current annealing led to partial recovery of the performance of two of the three lasers for which annealing was attempted. Recent results[1-3] on proton-implanted devices are summarized in a table.
\end{abstract}

\section{INTRODUCTION}

Vertical-cavity surface-emitting lasers (VCSELs)[4] can be directly modulated at rates in excess of $10 \mathrm{GHz}$, and can be used to transmit signals through space or in multimode optical fibers. Accordingly, they show great promise for use in optical interconnects and are expected to be useful in satellites and other systems that will be subjected to elevated levels of radiation. It is therefore of interest to determine the radiation levels at which performance changes occur, and to specify the nature of these changes. Additionally, it is desirable to determine the extent to which the performance can be restored by operating the lasers under various conditions. [5]

VCSELs fabricated at Sandia National Laboratories, designed to operate at $780 \mathrm{~nm}$ and $850 \mathrm{~nm}$ wavelengths have been irradiated thus far. The VCSELs are grown with Al$\mathrm{GaAs}$ or GaAs quantum wells. In the barriers and mirror stacks, the molar fraction of aluminum depends on the layer. The mirror layer interfaces have graded composition to decrease the series resistance of the structure. During fabrication, the current was laterally confined to the desired lasing region in two ways. Protons were implanted in some of the lasers, and selective oxidation[4] of AlGaAs layers confined the current in others. Figure 1 shows diagrams of the two types of lasers. In the proton implanted lasers, the light output regions are circular, with diameter $d$ ranging from $6 \mu \mathrm{m}$ to $16 \mu \mathrm{m}$.

Here, we emphasize the oxide-confined lasers, which operate at about $850 \mathrm{~nm}$ and have $5 \mathrm{GaAs}$ quantum wells. In the oxide confined lasers, the light output regions are square. Due to the sharp definition of the current aperture in the oxide lasers, they can be made with smaller areas

\footnotetext{
${ }^{1}$ This work was supported by United States Air Force Phillips Laboratory project funding.
}

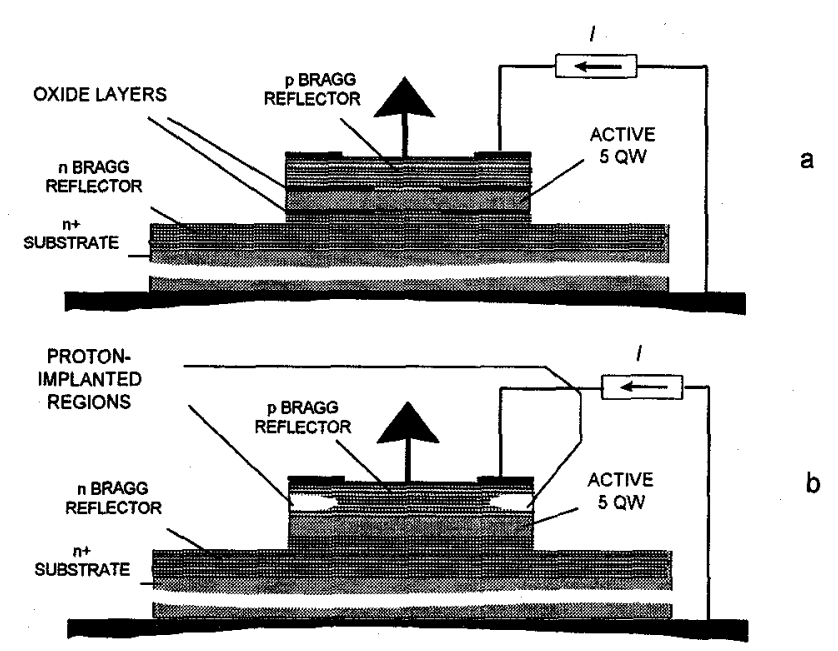

Fig. 1. Structure of VCSELs. The resonant cavity is vertical, with the mode confined between two Bragg reflectors made up of quarter-wave stacks of AlGaAs with the Al concentration varying between high and low values. In the center of a one-wavelengththick undoped region, there are $5 \mathrm{GaAs}$ quantum wells where there is optical gain. Two types of lasers were investigated, (a) lasers with the lateral current confinement provided by edgeoxidized layers with very high $\mathrm{Al}$ content, and (b) lasers with current apertures defined by proton implantation.

than are practical for the proton implanted lasers. The oxide VCSELs that we irradiated have smaller apertures than any of the proton implanted VCSELs that we exposed. For currents slightly above threshold, $(1-2 \mathrm{~mA})$, the measured near-field beam widths (full width between $e^{-2}$ intensity points) of five of the lasers varied from $3.3 \mu \mathrm{m}$ to $4.3 \mu \mathrm{m}$. At high current $(8-9 \mathrm{~mA})$, the widths varied from $3.9 \mu \mathrm{m}$ to $5.0 \mu \mathrm{m}$. Another feature that is unique to the oxideconfined lasers is the strain that is caused around the edges of the active region because a smaller volume is occupied by the oxide than the AlGaAs that was oxidized to form it. This strain may affect the formation and migration of defects.

We do not believe that oxide charging due to irradiation has much of an effect on the the performance of the lasers, since charge in the doped regions adjacent to the oxide layers can distribute itself to cancel the field. Moreover, the separation of the oxide layers is much smaller than the width of the conducting aperture. 
VCSELs exhibit thermal rollover of the light output power, with the power decreasing for currents above a certain level.[6] Operating a VCSEL with injected current values in the vicinity of the peak of the $L, I$ (light output power vs. current) curve will not damage it. In contrast, the $L, I$ curves of many edge emitting lasers are monotonically increasing and include straight sections, with the slope of a section depending on the number of operating modes. Thermal rollover may not occur within the practical range of operation of these lasers, with the upper limit on the optical power resulting from the occurrence of catastrophic optical damage to the laser output facet.[7]

In the gain medium of a radiation damaged semiconductor laser, nonradiative carrier recombination at radiationinduced defects competes with radiative recombination.[5] This is the dominant degradation mechanism in horizontalcavity lasers. Heat generation due to the added nonradiative recombination and carrier removal has a small influence on horizontal-cavity lasers ${ }^{2}$, but affects vertical-cavity lasers significantly. The density of longitudinal modes is great enough in horizontal-cavity lasers that the lasing wavelength at a given temperature is always within a small fraction of the gain curve width of the gain peak. On the other hand, due to the short cavity and the narrow-band high-reflectivity mirrors, only one longitudinal mode can operate in a VCSEL cavity, and the alignment of the cavity mode relative to the gain curve peak has a large influence on the operating efficiency. In a VCSEL, a temperature increase causes the cavity resonance and the gain peak to move to longer wavelengths. The shift of the gain curve is greater than the shift of the resonant wavelength, so the alignment of the cavity resonance with the gain curve changes with temperature. A VCSEL can even be designed so that its efficiency increases with temperature over a limited range. As a result of the existence of only a single low-loss longitudinal cavity mode, temperature affects the efficiency of a VCSEL more than that of a horizontal-cavity laser.

Carrier removal by radiation-induced defects is expected to have a greater influence on the efficiency of VCSELs than horizontal-cavity lasers because the doping profile of a VCSEL is the result of a delicate tradeoff. Increased doping reduces the series resistance across the multiple heterojunctions in the mirror stacks, but it also increases light absorption. [8]

\section{Experimental Procedure}

The VCSELs were irradiated with $4.5 \mathrm{MeV}$ protons at the microbeam facility at Sandia National Laboratories. The energy was high enough that the protons would pass well into the substrate before stopping. A TRIM[9] calculation indicated that the protons penetrated the laser

\footnotetext{
${ }^{2}$ We are considering horizontal cavity lasers in which no diffraction gratings interact with the modes. Distributed feedback lasers or lasers with distributed Bragg reflectors would have thermal properties more like those of VCSELs.
}

structure to a depth of $115 \mu \mathrm{m}$. Thus, the current could pass around the severe end-of-range damage because the lateral extent of the substrate is much greater than that of the irradiated region. The proton beam had $90 \%$ of its flux within a $2 \mu \mathrm{m}$ by $2 \mu \mathrm{m}$ square, and was rastered over a square in a pattern consisting of 512 by 512 pixels, with complete coverage of the square taking about $40 \mathrm{~ms}$. The dwell time on each pixel was about a factor of 10 times the time for moving between the pixels. Except within $2 \mu \mathrm{m}$ of the edges, the rastered region, which was 50 to 75 microns on a side, was very uniformly exposed. This resulted from a beam width which was at least 10 times the horizontal or vertical step size. The beam current fluctuated by about $10 \%$, but the large number of scans during exposure times of interest led to an averaged variation between pixels that was insignificant, less than $0.1 \%$ for a $1000 \mathrm{~s}$ exposure. Six VCSELs, all on a single die, were irradiated, nominally along a surface normal $\left( \pm 1^{\circ}\right)$. The normal to the emitting surface makes a $2^{\circ}$ angle with the $(100)$ crystal axis, tilted toward the (110) axis. The VCSELs were irradiated in a vacuum, and the lack of convective cooling affected their performance as shown by the lowered light output curves that were obtained immediately prior to exposure. When the lasers were irradiated, they had not been burned in. We burned in a control laser that was not irradiated, and its characteristics changed slightly. The peak output power decreased by $7 \%$, but the threshold current was unchanged

The lasers were not run during irradiation, except that after each interval of irradiation, the laser current was scanned, within about 1 second, from 0 to $11 \mathrm{~mA}$ to monitor the degradation. During the current scan, the output from the laser was measured, as was the voltage. The laser light was focused on a detector by a $36 \mathrm{~mm}$ diameter concave mirror with a $10.0 \mathrm{~mm}$ focal length. The proton beam passed through a $1.4 \mathrm{~mm}$ diameter hole which makes a $22.5^{\circ}$ angle with the normal to the mirror at its center. As discussed below, we believe that the detector received about $60 \%$ of the laser output light when it was measured during exposure.

\section{Results AND Discussion}

Figure 2 shows three $L, I$ (light-current) curves for one of the lasers (no. 1). The top $L, I$ curve was made with the laser in air, prior to irradiation. It shows thermal rollover which occurs mainly because the cavity resonance becomes increasingly misaligned with the peak of the gain curve as the temperature increases above a certain value. The middle $L, I$ curve, made in a vacuum prior to irradiation, has a lower peak value of the output light and a slightly higher threshold current, due to the lack of convective cooling. Because only part of the light was collected, we normalized the curves measured in vacuum so that the differential quantum efficiency of the pre-exposure curve matched that of the pre-exposure curve in air. ${ }^{3}$ This normalization gave

\footnotetext{
${ }^{3}$ Aside from the influence of operation in a vacuum, the shape of the curve might have been changed slightly by the fact that a smaller
} 


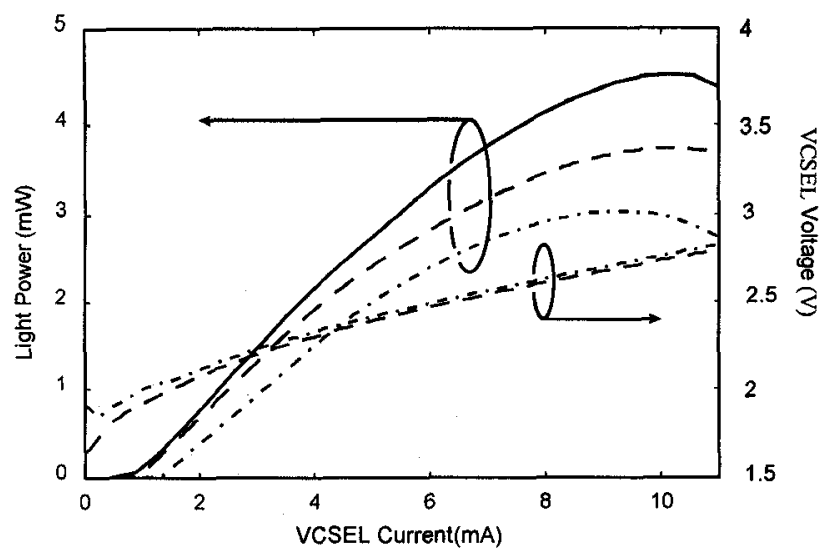

Fig. 2. Light-current and voltage-current curves for laser no. 1.

$60 \%$ for the collection efficiency of the laser light by the PIN diode. The bottom $L, I$ curve was taken after exposure to a proton fluence of $1.34 \times 10^{13} \mathrm{~cm}^{-2}$ and shows $19 \%$ peak-power degradation. After irradiation, the peakoutput current also became lower, which was typical of all the lasers.

Shown on the same figure are voltage curves taken in vacuum before and after exposure. Irradiation caused a slight increase in voltage (20-30 $\mathrm{mV}$ ) above threshold and larger increase with a region of negative resistance below threshold. This voltage increase contrasts with the characteristics of proton-irradiated horizontal-cavity lasers studied in Ref. 10 , for which shortened carrier lifetime led to an increase in the junction current for a given voltage, particularly for current well below threshold. Thus, damage in the undoped junction region of a laser diode causes a decrease in the voltage corresponding to a given current. Accordingly, the voltage increase we observed in the VCSELs must be a result of carrier removal from the doped regions. The added resistive heating degrades the performance by the mechanism described in the introduction and discussed in Ref. 6.

The threshold shifts of four of the lasers as a function of proton fluence are plotted on Fig. 3. The threshold shifts were obtained by fitting a straight line to a segment of each $L, I$ curve above the rapid increase in slope that signals the transition from spontaneous to stimulated emission. As long a segment as possible was selected, for which a straight line was a good approximation. The threshold current was given by the intersection of the fitted line with the $I$ axis. Evans et al. defined a constant relating the threshold shift of horizontal-cavity lasers to the fluence of irradiating protons, $\Phi,[11]$

$$
I_{t h}(\Phi) / I_{t h}(0)=1+\kappa \Phi
$$

fraction of the light from the second order transverse modes was collected than from the first order mode. The fraction of the power in higher order modes varies with the current.

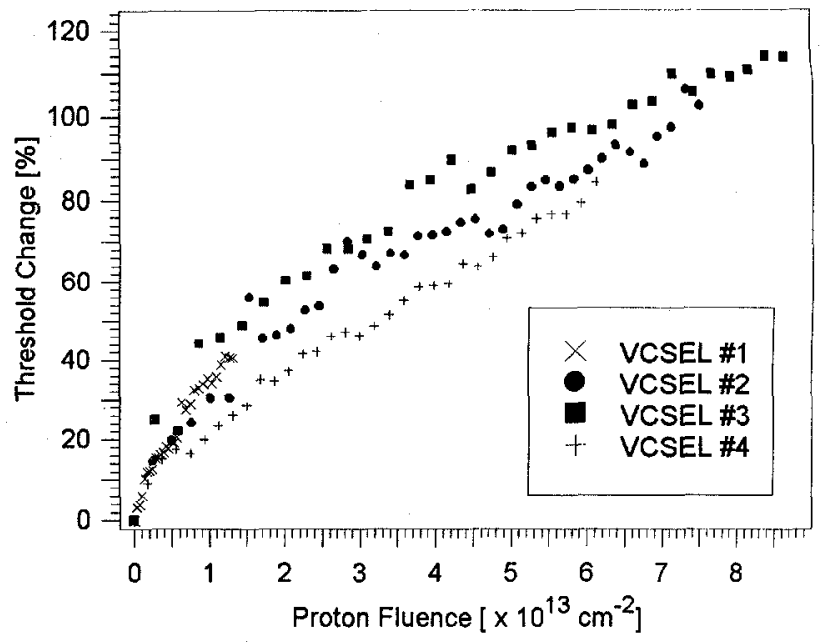

Fig. 3. Threshold shifts of the four lasers with the lowest preirradiation threshold currents, as a function of proton fluence.

For these VCSELs, the threshold shift is not proportional to the radiation fluence, but we can define a function, $\kappa(\Phi)$, that satisfies Eq. 1. For $\Phi=2 \times 10^{13} \mathrm{~cm}^{-2}$, $\kappa=2.4 \times 10^{-14} \mathrm{~cm}^{2} \pm 25 \%$, and for $\Phi=8 \times 10^{13} \mathrm{~cm}^{-2}$, $\kappa=1.4 \times 10^{-14} \mathrm{~cm}^{2}$. The value obtained by Evans et al. for edge-emitting lasers that were operated during irradiation was $\kappa=7.4 \pm 1.7 \times 10^{-14} \mathrm{~cm}^{2}$. Thus, the proton fluence that caused a threshold shift of $50 \%$ or more for our VCSELs is several times larger than the fluence that caused the same shift in the $\operatorname{In}_{0.2} \mathrm{Ga}_{0.8} \mathrm{As}$-quantum-well lasers that were evaluated by Evans et al. ${ }^{4}$

We have recently evaluated the radiation hardness of VCSELs, also grown and fabricated at Sandia National Laboratories, with the conducting region surrounded by proton implanted regions rather than selectively oxidized regions.[1-3] The dominant result of radiation damage in those lasers was a decrease in the peak output power. The peak-output current also became lower. For the majority of the lasers, no increase in the threshold current occurred for proton fluence levels that caused substantial degradation, around $10-50 \%$, of the peak power. $[1,2]$ With one exception, the proton-implanted VCSELs were operated well above threshold during irradiation.

In contrast to the proton implanted lasers, the oxideconfined VCSELs showed threshold increases for any fluence level that substantially reduced (by about $20 \%$ ) the

\footnotetext{
'The protons in Evans' experiment arrived at the quantum well with $3.0 \mathrm{MeV}$ of energy. The NIEL obtained for this energy in GaAs is $46 \%$ higher than for $4.5 \mathrm{MeV}$ protons, and the presence of indium is not believed to change its value by much.[11] For identical lasers, one would expect $\kappa$ to be $46 \%$ higher for $3.0 \mathrm{MeV}$ protons than for 4.5 $\mathrm{MeV}$ protons. The stability of defects, or the carrier recombination rate at them, could, however, be changed by the presence of indium and by the strain it induces. Therefore, the materials as well as the structures probably influenced the relative sensitivity of the two types of lasers.
} 
TABLE I

PEAK-POWER REDUCTION

\begin{tabular}{|c|c|r|l|r|l|l|c|}
\hline$\lambda(\mathrm{nm})$ & $d(\mu \mathrm{m})$ & $\Phi\left(\times 10^{13} \mathrm{~cm}^{-2}\right)$ & $D(\%)$ & $\Phi\left(\times 10^{13} \mathrm{~cm}^{-2}\right)$ & $D(\%)$ & oxide? & notes \\
\hline \hline 850 & 4 & 1.0 & 15 & 1.3 & 19 & yes & $\dagger$ \\
\hline 850 & 4 & 2.0 & 25 & 8.7 & 59 & yes & $\dagger$ \\
\hline 850 & 4 & 2.1 & 33 & 7.5 & 58 & yes & $\dagger$ \\
\hline 850 & 4 & 2.1 & 49 & 6.1 & 64 & yes & $\dagger$ \\
\hline 850 & 4 & 2.1 & 29 & 5.5 & 56 & yes & $\dagger$ \\
\hline 850 & 4 & & & 3.5 & 59 & yes & $\dagger$ \\
\hline 780 & 16 & & & 1.8 & 8 & no & $\ddagger$ \\
\hline 780 & 16 & & & 1.1 & 14 & no & \\
\hline 780 & 16 & 0.30 & 11 & 1.3 & 26 & no & \\
\hline 780 & 16 & 1.1 & 38 & 2.1 & 60 & no & $\dagger^{*}$ \\
\hline 780 & 6 & 2.0 & 23 & 3.8 & 37 & no & \\
\hline 780 & 6 & & & 1.9 & 29 & no & \\
\hline 850 & 11 & 0.68 & 17 & 1.4 & 36 & no & \\
\hline 850 & 11 & & & 2.5 & 46 & no & \\
\hline
\end{tabular}

$\dagger$ Not operated during irradiation.

$\ddagger$ Proton energy was $2.0 \mathrm{MeV}$. NIEL (GaAs) $=1.6 \times 10^{2} \mathrm{kev} / \mathrm{cm}$

*The pre-irradiation $L, I$ curve stopped just short of the maximum output power, and we used its maximum in place of the actual maximum.

peak power. This corresponds more closely with the symptoms of proton irradiation damage of horizontal-cavity lasers. This may not show a fundamental difference between the response of the two types of VCSELs, because the proton implanted lasers were operated well above threshold during irradiation, with one exception, while the oxideconfined lasers were unbiased. The only proton-implanted laser that showed a significant threshold increase due to irradiation was the unbiased one. Therefore, further work is necessary to determine whether there is a qualitative difference between the responses of the two types of lasers.

Three of the oxide-confined lasers were operated after exposure, to determine whether forward-current annealing would occur. Two of the lasers annealed, and the third laser, which was subjected to the highest proton fluence, rapidly deteriorated and ceased to lase. The annealing history of one of the lasers (no. 1) is shown in Fig. 4. The annealing current was $4.00 \mathrm{~mA}$ and the threshold recovered from $1.38 \mathrm{~mA}$ to $1.17 \mathrm{~mA}$ in 10 hours. The pre-irradiation threshold current was $0.93 \mathrm{~mA}$. Except for the first three points, a decaying exponential curve for the recovery fits Fig. 4. The e-folding time for the threshold recovery is $1.3 \times 10^{4} \mathrm{~s}$. The initial recovery is too rapid to fit this time constant, possibly indicating the existence of two types of defects, one of which anneals very rapidly when lasing current is applied.

Table 1 gives the peak power decrease, $D$, suffered by the lasers. We have included data for VCSELs with proton implant current confinement, taken from Refs. 1 and 2 . During irradiation, the oxide-confined VCSELs were unbiased and all but one of the proton implanted VCSELs

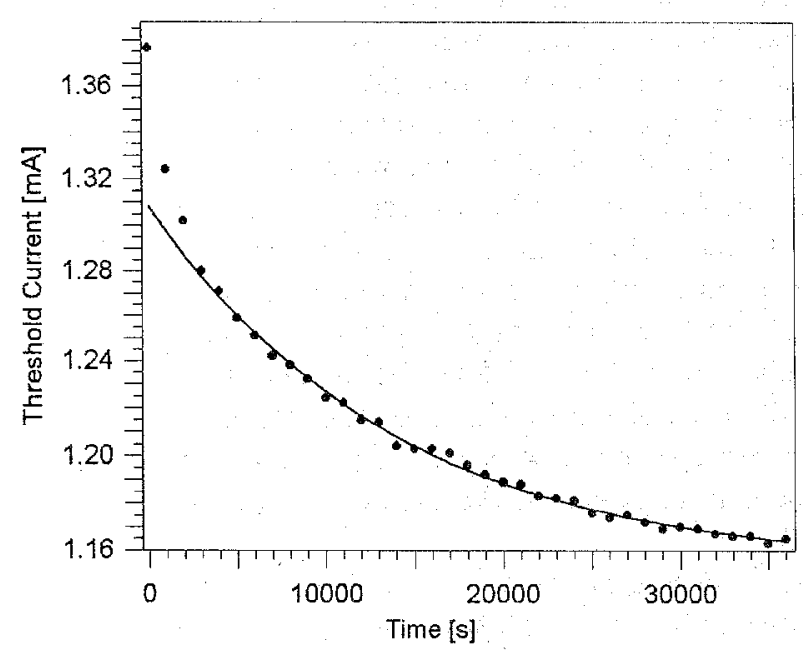

Fig. 4. Annealing history of an oxide-confined VCSEL, laser no, 1 that was exposed to a fluence of $1.34 \times 10^{13}$ protons $/ \mathrm{cm}^{2}$.

were operated well above threshold. Significant changes in the performance of the oxide-confined VCSELs began to occur at fluences around $1 \times 10^{13}$ protons $/ \mathrm{cm}^{2}$. This fluence can be expressed in terms of the dose received in the quantum wells. For the $850 \mathrm{~nm}$ lasers, the dose was $6.9 \mathrm{Mrad}(\mathrm{GaAs})$ per $10^{13}$ protons $/ \mathrm{cm}^{2}$. The dose is not, however, a good measure of the density of induced defects that cause VCSEL performance degration. The density of performance-degrading defects formed in electronic semiconductor devices is proportional to the non-ionizing en- 
ergy loss (NIEL), for several types of ionizing radiation over a wide range of particle energy.[12] The $4.5 \mathrm{MeV}$ protons have a NIEL $=73 \mathrm{keV} / \mathrm{cm}$ in the GaAs quantum wells, read from a graph in Ref. 12 .

\section{CONCLUSIONS}

Degradation should be considered in designing a system including the type of oxide-confined VCSELs that are the subject of this paper for a fluence of $4.5 \mathrm{MeV}$ protons on the order of $\Phi=10^{13} \mathrm{~cm}^{-2}$, or an irradiation level giving equivalent lattice damage. At $\Phi=2 \times 10^{13} \mathrm{~cm}^{-2}$, the decrease in the peak output power was in the range between $25 \%$ and $40 \%$, and the threshold current shifts varied from $35 \%$ to $60 \%$.

Annealing data were taken for one of the lasers that was exposed to $\Phi=1.34 \times 10^{13} \mathrm{~cm}^{-2}$. Its threshold current increased from $0.93 \mathrm{~mA}$ to $1.38 \mathrm{~mA}$ due to irradiation. After $4.0 \mathrm{~mA}$ forward current was applied for 10 hours, the threshold current recovered to $1.16 \mathrm{~mA}$. In contrast to data taken for edge-emitting lasers, the VCSEL data show increased voltage due to carrier removal from the doped regions. This increase results partially from the relatively low dopant density (prior to irradiation) in parts of the Bragg reflector stacks, and partially from the small transverse area of the conducting region.

\section{ACKNOWLEDGEMENTS}

We wish to thank Y. F. Zhao for providing us with a preprint of Ref. 10, and we are grateful to Scot Sumner of S\&S Optical Fabrication in Tucson, Az. for drilling the hole through the concave light-focusing mirror.

\section{REFERENCES}

[1] R.F. Carson et al., "Surface emitting laser technology and its application to the space radiation environment," in Advancement of Photonics for Space, E. W. Taylor, Editor, Bellingham, WA: SPIE vol. CR66, 1997, pp. 121-151.

[2] H. Schöne, R. F. Carson, A. H. Paxton, and E. W. Taylor, "AlGaAs VCSEL array responses to proton irradiation", accepted for publication in IEEE Photonics Technology Letters.
[3] E. W. Taylor, A.H. Paxton, R. F. Carson, H. Schöne, J. Bristow, J. Lehman, and M. Hibbs-Brenner, "En. vacuo responses of of an AlGaAs vertical cavity surface emitting laser irradiated by a $4.5 \mathrm{MeV}$ ion microbeam," presented at the conference on Radiations and their Effects on Devices and Systems, Cannes, France (18 September 1997), submitted for publication.

[4] K. D. Choquette, K. L. Lear, R. P. Schneider, Jr., K. M. Geib, J.J. Figiel, and Robert Hull, "Fabrication and performance of selectively oxidized vertical-cavity lasers," IEEE Photon. Technol. Lett., vol. 7, pp. 1237$1239,1995$.

[5] C. E. Barnes, "Effects of $\mathrm{CO}^{60}$ irradiation on epitaxial GaAs laser diodes," Phys. Rev. B, vol. 1, pp. 4735-4747, 1970.

[6] G. Hasnain, K. Tai, L. Yang, Y.H. Wang, R. J. Fischer, J. D. Wynn, B. Weir, N. K. Dutta, and A. Y. Cho, "Performance of gain-guided surface emitting lasers with semiconductor distributed Bragg reflectors," IEEE J. Quantum Electron., vol. 27, pp. 1377-1385, 1991.

[7] N. W. Carlson, Monolithic Diode-Laser Arrays, Berlin: Springer-Verlag, 1994, pp. 63-66.

[8] K. L. Lear, R. P. Schneider, K. D. Choquette, S. P. Kilcoyne, J. J. Figuel, and J.C. Zolper, "Vertical cavity surface emitting lasers with $21 \%$ efficiency by metalorganic vapor phase epitaxy," IEEE Photon. Technol. Lett., vol. 6, pp. 1053-1055, 1994.

[9] J. F. Ziegler, J. P. Biersack, and U. Littmark, The Stopping Range of Ions in Solids, New York: Pergamon Press, 1985.

[10] Y. F. Zhao, A. R. Patwary, R. D. Schrimpf, M. A. Neifeld, and K. F. Galloway, "200 MeV proton damage effects on multi-quantum well laser diodes," IEEE Trans. Nucl. Sci., vol. 44, December, 1997.

[11] B.D. Evans, H. E. Hager, and B. W. Hughlock, "5.5$\mathrm{MeV}$ proton irradiation of a strained quantum-well Laser diode and a multiple quantum-well broad-band LED," IEEE Trans. Nucl. Sci., vol. 40, pp. 1645-1654, 1993.

[12] G.P. Summers, E. A. Burke, P. Shapiro, S. R. Messenger, and R. J. Walters, "Damage correlations in semiconductors exposed to gamma, electron, and proton irradiations," IEEE Trans. Nucl. Sci., vol. 40, pp. 13721379, 1993. 